\title{
REDSHIFT DETERMINATION AND CO LINE EXCITATION MODELING FOR THE MULTIPLY LENSED GALAXY HLSW-01
}

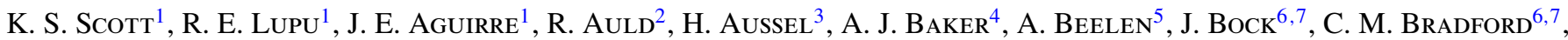 \\ D. Brisbin ${ }^{8}$, D. Burgarella ${ }^{9}$, J. M. Carpenter ${ }^{6}$, P. Chanial $^{3}$, S. C. Chapman ${ }^{10}$, D. L. Clements ${ }^{11}$, A. Conley $^{12}$, \\ A. Cooray ${ }^{6,13}$, P. Cox ${ }^{14}$, C. D. Dowell ${ }^{6,7}$, S. EAles $^{2}$, D. Farrah ${ }^{15}$, A. Franceschini ${ }^{16}$, D. T. Frayer ${ }^{17}$, R. Gavazzi ${ }^{18}$,

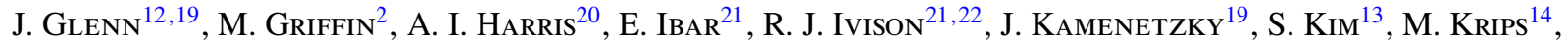 \\ P. R. Maloney ${ }^{12}$, H. Matsuhara ${ }^{23}$, A. M. J. Mortier ${ }^{11}$, E. J. Murphy ${ }^{6,24}$, B. J. Naylor ${ }^{7}$, R. Neri $^{14}$, H. T. NguYen $^{6,7}$,

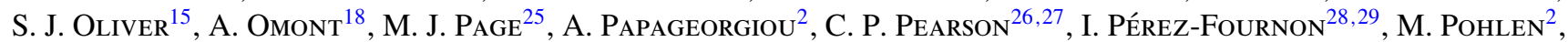

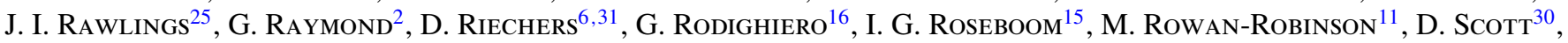 \\ N. Seymour ${ }^{25}$, A. J. Smith ${ }^{15}$, M. Symeonidis ${ }^{25}$, K. E. Tugwell ${ }^{25}$, M. VACCARi ${ }^{16}$, J. D. Vieira ${ }^{6}$ L. VigrouX $^{18}$, L. WAnG $^{15}$, \\ G. WRIGHT ${ }^{21}$, AND J. ZMUIDZINAS ${ }^{6,7}$ \\ ${ }^{1}$ Department of Physics and Astronomy, University of Pennsylvania, Philadelphia, PA 19104, USA \\ 2 School of Physics and Astronomy, Cardiff University, Queens Buildings, The Parade, Cardiff, CF24 3AA, UK \\ ${ }^{3}$ Laboratoire AIM-Paris-Saclay, CEA/DSM/Irfu-CNRS-Université Paris Diderot, CE-Saclay, pt courrier 131, F-91191 Gif-sur-Yvette, France \\ ${ }^{4}$ Department of Physics and Astronomy, Rutgers, The State University of New Jersey, 136 Frelinghuysen Road, Piscataway, NJ 08854, USA \\ ${ }^{5}$ Institut d'Astrophysique Spatiale (IAS), bâtiment 121, Université Paris-Sud 11 and CNRS (UMR 8617), 91405 Orsay, France \\ ${ }^{6}$ California Institute of Technology, 1200 East California Boulevard, Pasadena, CA 91125, USA \\ ${ }^{7}$ Jet Propulsion Laboratory, 4800 Oak Grove Drive, Pasadena, CA 91109, USA \\ ${ }^{8}$ Space Science Building, Cornell University, Ithaca, NY 14853-6801, USA \\ ${ }^{9}$ Laboratoire d'Astrophysique de Marseille, OAMP, Université Aix-marseille, CNRS, 38 rue Frédéric Joliot-Curie, 13388 Marseille Cedex 13, France \\ ${ }^{10}$ Institute of Astronomy, University of Cambridge, Madingley Road, Cambridge CB3 OHA, UK \\ ${ }^{11}$ Astrophysics Group, Imperial College London, Blackett Laboratory, Prince Consort Road, London SW7 2AZ, UK \\ ${ }^{12}$ Center for Astrophysics and Space Astronomy 389-UCB, University of Colorado, Boulder, CO 80309, USA \\ ${ }^{13}$ Department of Physics \& Astronomy, University of California, Irvine, CA 92697, USA \\ ${ }^{14}$ Institut de Radioastronomie Millimétrique, 300 Rue de la Piscine, Domaine Universitaire, 38406 Saint Martin d'Hères, France \\ ${ }^{15}$ Astronomy Centre, Department of Physics \& Astronomy, University of Sussex, Brighton BN1 9QH, UK \\ ${ }^{16}$ Dipartimento di Astronomia, Università di Padova, vicolo Osservatorio 3, 35122 Padova, Italy \\ ${ }^{17}$ NRAO, P.O. Box 2, Green Bank, WV 24944, USA \\ ${ }^{18}$ Institut d'Astrophysique de Paris, UMR 7095, CNRS, UPMC Univ. Paris 06, 98 bis boulevard Arago, F-75014 Paris, France \\ ${ }^{19}$ Department of Astrophysical and Planetary Sciences, CASA 389-UCB, University of Colorado, Boulder, CO 80309, USA \\ ${ }^{20}$ Department of Astronomy, University of Maryland, College Park, MD 20742-2421, USA \\ ${ }^{21}$ UK Astronomy Technology Centre, Royal Observatory, Blackford Hill, Edinburgh EH9 3HJ, UK \\ ${ }^{22}$ Institute for Astronomy, University of Edinburgh, Royal Observatory, Blackford Hill, Edinburgh EH9 3HJ, UK \\ ${ }^{23}$ Institute for Space and Astronautical Science, Japan Aerospace and Exploration Agency, Sagamihara, Kanagawa 229-8510, Japan \\ ${ }^{24}$ Infrared Processing and Analysis Center, MS 100-22, California Institute of Technology, JPL, Pasadena, CA 91125, USA \\ ${ }^{25}$ Mullard Space Science Laboratory, University College London, Holmbury St. Mary, Dorking, Surrey RH5 6NT, UK \\ ${ }^{26}$ RAL Space, Rutherford Appleton Laboratory, Chilton, Didcot, Oxfordshire OX11 0QX, UK \\ ${ }^{27}$ Institute for Space Imaging Science, University of Lethbridge, Lethbridge, Alberta T1K 3M4, Canada \\ ${ }^{28}$ Instituto de Astrofísica de Canarias (IAC), E-38200 La Laguna, Tenerife, Spain \\ ${ }^{29}$ Departamento de Astrofísica, Universidad de La Laguna (ULL), E-38205 La Laguna, Tenerife, Spain \\ ${ }^{30}$ Department of Physics \& Astronomy, University of British Columbia, 6224 Agricultural Road, Vancouver, BC V6T 1Z1, Canada \\ Received 2011 February 14; accepted 2011 March 14; published 2011 April 29
}

ABSTRACT

We report on the redshift measurement and CO line excitation of HERMES J105751.1+573027 (HLSW-01), a strongly lensed submillimeter galaxy discovered in Herschel/SPIRE observations as part of the Herschel Multi-tiered Extragalactic Survey (HerMES). HLSW-01 is an ultra-luminous galaxy with an intrinsic far-infrared luminosity of $L_{\mathrm{FIR}}=1.4 \times 10^{13} L_{\odot}$, and is lensed by a massive group of galaxies into at least four images with a total magnification of $\mu=10.9 \pm 0.7$. With the $100 \mathrm{GHz}$ instantaneous bandwidth of the Z-Spec instrument on the Caltech Submillimeter Observatory, we robustly identify a redshift of $z=2.958 \pm 0.007$ for this source, using the simultaneous detection of four CO emission lines $(J=7 \rightarrow 6, J=8 \rightarrow 7, J=9 \rightarrow 8$, and $J=10 \rightarrow 9$ ). Combining the measured line fluxes for these high- $J$ transitions with the $J=1 \rightarrow 0, J=3 \rightarrow 2$, and $J=5 \rightarrow 4$ line fluxes measured with the Green Bank Telescope, the Combined Array for Research in Millimeter Astronomy, and the Plateau de Bure Interferometer, respectively, we model the physical properties of the molecular gas in this galaxy. We find that the full $\mathrm{CO}$ spectral line energy distribution is described well by warm, moderate-density gas with $T_{\text {kin }}=86-235 \mathrm{~K}$ and $n_{\mathrm{H}_{2}}=(1.1-3.5) \times 10^{3} \mathrm{~cm}^{-3}$. However, it is possible that the highest- $J$ transitions are tracing a small fraction of very dense gas in molecular cloud cores, and two-component models that include a warm/dense molecular gas phase with $T_{\text {kin }} \sim 200 \mathrm{~K}, n_{\mathrm{H}_{2}} \sim 10^{5} \mathrm{~cm}^{-3}$ are also consistent with these data. Higher signal-to-noise measurements of the $J_{\text {up }} \geqslant 7$ transitions with high spectral resolution, combined with high spatial resolution $\mathrm{CO}$ maps, are needed to improve our understanding of the gas excitation, morphology, and dynamics of this interesting high-redshift galaxy.

Key words: galaxies: high-redshift - galaxies: individual (HERMES J105751.1+573027) - galaxies: starburst submillimeter: galaxies 


\section{INTRODUCTION}

Galaxies selected at submillimeter (submm) and millimeter $(\mathrm{mm})$ wavelengths (hereafter SMGs) are predominantly highredshift (1.5 $\lesssim z \lesssim 3.5)$ systems passing through an important, intense starburst phase of their evolution, with typical star formation rates (SFRs) $>100 M_{\odot} \mathrm{yr}^{-1}$ (e.g., Blain et al. 2002; Chapman et al. 2005). Over the past 13 years, a large number of deep, wide-area surveys at $\lambda=850-1200 \mu \mathrm{m}$ have been conducted from ground-based telescopes (e.g., Scott S. E., et al. 2002; Greve et al. 2004; Coppin et al. 2006; Bertoldi et al. 2007; Perera et al. 2008; Weiß et al. 2009b; Austermann et al. 2010; Scott, K. S., et al. 2010). These wavelengths sample the Rayleigh-Jeans tail of thermal dust emission, where this dust is heated by ultraviolet (UV) light from intense star formation or active galactic nuclei (AGNs). Owing to a strong negative $k$-correction, galaxies with the same bolometric luminosity (a proxy for SFR) that are selected at $\lambda \gtrsim 500 \mu \mathrm{m}$ are equally detectable for $1 \lesssim z \lesssim 10$ in a flux-limited survey. However, the relative ease of detecting a significant number of SMGs in deep, wide-area surveys is countered by the time-consuming multiwavelength follow-up necessary to derive information on their redshifts, SFRs and star formation efficiencies, morphologies, and dynamics, and on the importance of AGNs in these systems (e.g., Chapman et al. 2005; Pope et al. 2006; Younger et al. 2007, 2009; Biggs et al. 2010).

Measuring redshifts for a large number of SMGs is necessary for determining their contribution to the cosmic star formation history (Chapman et al. 2005; Aretxaga et al. 2007), and is crucial for carrying out detailed studies of a representative sample of this population. Obtaining redshifts through optical spectroscopy is difficult, given the poor positional accuracy of most SMGs (arising from the low resolution of ground-based submm $/ \mathrm{mm}$ telescopes) and their extreme dust obscuration. The most direct redshift measurement for SMGs is through the detection of the rotational transitions of carbon monoxide (CO), since SMGs contain large reservoirs of molecular gas $\left(\sim 10^{10}-10^{11} M_{\odot}\right.$; Greve et al. 2005; Tacconi et al. 2006, 2008). Until very recently - with the exception of three blind CO redshift detections (Weiß et al. 2009a; Swinbank et al. 2010; Lestrade et al. 2010) - CO measurements of SMGs had been limited to sources with known, optically determined redshifts (e.g., Frayer et al. 1998, 1999, 2008; Ivison et al. 2001; Downes \& Solomon 2003; Genzel et al. 2003; Neri et al. 2003; Sheth et al. 2004; Kneib et al. 2005; Greve et al. 2005; Tacconi et al. 2006; Chapman et al. 2008; Bothwell et al. 2010; Engel et al. 2010), since the limited bandwidth and sensitivity of most receivers precluded a blind search for redshifted CO lines.

Two recent developments have spurred the rapid growth of the number of SMGs with blind CO redshift detections over the past year. The first is the availability of sensitive, widebandwidth receivers designed specifically for the detection of one or more CO lines. These include Z-Spec, a grating spectrometer with 160 silicon-nitride micro-mesh bolometers operating from 190 to $310 \mathrm{GHz}$ (Naylor et al. 2003; Earle et al. 2006; Bradford et al. 2009). For $z>0.5$, at least two CO transitions are redshifted into the Z-Spec bandpass, allowing for a robust redshift determination for galaxies at $z \lesssim 3$ (or higher, provided that the CO $J_{\text {up }} \gtrsim 8$ transitions are excited). The second development is the recent detection of extremely bright SMGs with apparent far-infrared (FIR) luminosities $\gtrsim 10^{14} L_{\odot}$.

\footnotetext{
${ }^{31}$ Hubble Fellow.
}

Although a few discoveries of such extreme SMGs have been made in small-scale mapping surveys (Swinbank et al. 2010; Ikarashi et al. 2010), large area (10-200 $\left.\mathrm{deg}^{2}\right)$ surveys carried out with the South Pole Telescope (SPT) at $1.4 \mathrm{~mm}$ (Vieira et al. 2010), the Atacama Cosmology Telescope at 1-2 mm, and with SPIRE (250-500 $\mu \mathrm{m}$; Griffin et al. 2010) on the Herschel Space Observatory (Pilbratt et al. 2010; Eales et al. 2010; Oliver et al. 2010) are uncovering a large number of these extreme SMGs, and are thus providing a sizable number of very bright targets that are ideal for follow-up $\mathrm{CO}$ measurements. Five of these extremely bright SMGs that were detected in the Herschel-ATLAS Science Demonstration Phase data were recently targeted for blind $\mathrm{CO}$ measurements with Z-Spec and Zpectrometer on the Green Bank Telescope (GBT), and all five redshifts were successfully measured (Lupu et al. 2010; Frayer et al. 2011). These redshifts have been key to demonstrating that these extremely bright SMGs are strongly lensed by intervening foreground galaxies (Negrello et al. 2010). With a growing number of strongly lensed SMGs being uncovered with Herschel/SPIRE and the SPT, the number of blind CO redshift measurements is expected to grow considerably, even before full operations of the Atacama Large Millimeter/submillimeter Array (ALMA) are underway.

The CO line fluxes constrain the physical properties of molecular gas in a galaxy, including the total molecular gas mass as well as temperature and density. Since estimates of physical conditions depend on the relative line strengths, it is necessary to sample the full spectral line energy distribution (SLED) from the low- to high- $J$ transitions in order to place useful constraints on the gas properties. While most galaxies at both low and high redshifts have only been detected in 1-2 CO lines (Solomon \& Vanden Bout 2005; Greve et al. 2005; Tacconi et al. 2006, 2008; Harris et al. 2010; Ivison et al. 2010b; Aravena et al. 2010; Wang et al. 2010), a growing number of galaxies have been observed in $\geqslant 3$ transitions in recent years. These include ground-based observations of galaxies at both low and high redshifts (Weiß et al. 2005a, 2007a, 2007b; Riechers et al. 2006; Ao et al. 2008; Bradford et al. 2009; Papadopoulos et al. 2010a, 2010b; Carilli et al. 2010; Riechers et al. 2010; Lestrade et al. 2010; Danielson et al. 2011) as well as recent Herschel SPIRE/Fourier-transform spectrometer (FTS; Panuzzo et al. 2010; van der Werf et al. 2010) and HIFI (Loenen et al. 2010) observations of nearby starbursts and active galaxies. For many, the full CO SLED is described well by a single component with warm, dense gas typical of starforming regions, with kinetic temperatures of $T_{\text {kin }} \sim 30-100 \mathrm{~K}$ and densities of $n_{\mathrm{H}_{2}} \sim 10^{3.5}-10^{6} \mathrm{~cm}^{-3}$. However, those with the best sampled SLEDs, spanning the full run of the rotational transition ladder, often require multiple gas phases to explain the observed line fluxes, with a warm/dense phase required to excite the mid- to high- $J$ transitions, and extended, cold lowexcitation gas that contributes significantly to the $J_{\text {up }} \lesssim 2$ line fluxes (Ward et al. 2003; Carilli et al. 2010; Danielson et al. 2011; Panuzzo et al. 2010; van der Werf et al. 2010). Several studies have found that most SMGs show an excess in the CO $J=1 \rightarrow 0$ line luminosity relative to the higher- $J$ transitions (Harris et al. 2010; Frayer et al. 2011), and high-resolution mapping of $\mathrm{CO}$ in a number of these high-redshift galaxies reveals that the $J=1 \rightarrow 0$ line traces a more extended gas reservoir than that traced by $J_{\text {up }} \gtrsim 3$ transitions (Ivison et al. 2010a).

In this paper, we present the $\mathrm{CO}$ redshift measurement and excitation modeling of HERMES J105751.1+573027 (hereafter HLSW-01), a multiply imaged SMG discovered in 
Science Demonstration Phase Herschel/SPIRE data as part of the Herschel Multi-tiered Extragalactic Survey (HerMES ${ }^{32}$; Oliver et al. 2010; S. J. Oliver et al. 2011, in preparation). High-resolution imaging at $880 \mu \mathrm{m}$ with the Submillimeter Array (SMA) reveals at least four images separated by $\sim 9^{\prime \prime}$, and higher resolution $K_{p}$-band imaging shows strong lensing arcs. The lensing model (Gavazzi et al. 2011, hereafter G11) suggests that HLSW-01 is lensed by a massive group of galaxies, with a total gravitational magnification of $\mu=10.9 \pm 0.7$. When corrected for the magnification, this galaxy is found to be a very bright ultra-luminous infrared galaxy (ULIRG) with an FIR luminosity of $L_{\mathrm{FIR}}=(1.43 \pm 0.09) \times 10^{13} L_{\odot}$ and an implied SFR of $2460 \pm 160 M_{\odot} \mathrm{yr}^{-1}$ (Conley et al. 2011, hereafter C11). The dust continuum spectral energy distribution (SED) is warm with a dust temperature of $T_{\mathrm{d}} \approx 88 \mathrm{~K}$ determined from a simple onecomponent-modified blackbody model (C11). Combined with a radio flux density in excess of that expected from the FIR to radio correlation, there is tentative evidence that this source harbors a bolometrically important AGN, in which case the SFR inferred from $L_{\text {FIR }}$ is overestimated.

This paper is organized as follows: we discuss the Z-Spec observations and data reduction for HLSW-01 in Section 2; in Section 3, we describe the redshift determination and line flux measurements from the Z-Spec data; we describe the $\mathrm{CO}$ excitation modeling in Section 4, and discuss the molecular gas properties in Section 5. We summarize these results in Section 6. We assume a flat, $\Lambda$ CDM cosmology with $\Omega_{\mathrm{M}}=0.3, \Omega_{\Lambda}=0.7$, and $H_{0}=70 \mathrm{~km} \mathrm{~s}^{-1} \mathrm{Mpc}^{-1}$ throughout this paper.

\section{Z-SPEC OBSERVATIONS AND DATA REDUCTION}

We carried out the Z-Spec observations of HLSW-01 at the Caltech Submillimeter Observatory (CSO) from 2010 March 9 to May 12 under generally good to excellent observing conditions, with a zenith opacity at $225 \mathrm{GHz}$ (monitored by the CSO tau meter) ranging over $\tau_{225}=0.03$ to 0.1 , with $\tau_{225} \leqslant 0.06$ for $80 \%$ of the observations. The beam size ranges from $25^{\prime \prime}$ to $40^{\prime \prime}$ (full width at half-maximum) over the Z-Spec bandpass. The data were taken using the standard "chop-and-nod" mode in order to estimate and subtract the atmospheric signal from the raw data. The secondary mirror was chopped on- and offsource at a rate of $1.6 \mathrm{~Hz}$, with a chop throw of $90^{\prime \prime}$ while stepping through a four-position nod cycle, integrating for $20 \mathrm{~s}$ at each nod position. We checked the pointing every $2-4 \mathrm{hr}$ by observing quasars and other bright targets located close in elevation to HLSW-01, making small (typically $\lesssim 10^{\prime \prime}$ ) adjustments to the telescope pointing model in real time. The total integration time (including the time spent in the off-source position during the nod cycle, but excluding all other overheads) was $22.9 \mathrm{hr}$.

We analyze the data using customized software in the same manner as described in Bradford et al. (2009). For each channel, the nods are calibrated and averaged together, weighted by the inverse square of the noise. Absolute calibration is determined by frequent ( $\sim 1$ per night) observations of Mars (Wright 2007) and Neptune. ${ }^{33}$ The flux density of Mars (proportional to $v^{2}$ from 190 to $310 \mathrm{GHz}$ ) decreased with apparent size from 800 Jy to $270 \mathrm{Jy}$ at $240 \mathrm{GHz}$ over the course of the Z-Spec Spring 2010 observing season, while the $240 \mathrm{GHz}$ flux density of Neptune was 16 Jy during this run. We use a total of 42 planet observations taken throughout the observing run to build a model

\footnotetext{
32 hermes.sussex.ac.uk

$33 \mathrm{http} / / /$ sma1.sma.hawaii.edu/planetvis.html
}

of the flux conversion factor (from voltage to flux density) as a function of operating (DC) voltage for each detector separately (Bradford et al. 2009). Since the DC voltage depends on a combination of the bath temperature and the total optical loading on the detectors, we use these curves to determine appropriate calibration factors to apply to each nod individually. Based on the rms deviations of the planet measurements from the bestfit curves, the channel calibration uncertainties are $4 \%-9 \%$, excluding the lowest frequencies for which a good model of the atmosphere is hindered by the $183 \mathrm{GHz}$ atmospheric water line. These errors are propagated through the data reduction and integrate down as the square root of the number of nods included in the average.

Since blind CO line detections with Z-Spec require small channel-to-channel variations, we determine small bandpass corrections to the calibration for each channel from continuum measurements of bright $\mathrm{mm}$ sources. We carried out a total of 67 observations of bright continuum sources $(1 \mathrm{~mm}$ flux density, $S_{1.1}>1 \mathrm{Jy}$ ), including J1055+018, J1637+574, J0854+201, 3C273, 3C279, and 3C345. Each was reduced using the calibration determined from the planet observations as described in the previous paragraph. We then fit a power law to the continuum, separately for each observation, and for each channel compute a multiplicative correction factor to apply to the observed spectrum. We exclude the measurements from the 12 lowest frequency channels $(v \leqslant 190 \mathrm{GHz}$, contaminated by the atmospheric water line) and from the $232.3 \mathrm{GHz}$ channel (known to be unstable) in the continuum fits. We then average over all observations to determine a single correction factor for each channel. These bandpass corrections are small (ranging from 0.96 to 1.06$)$, with little variation $(\leqslant 5 \%)$ over all observations. We apply these small correction factors to the calibrated data to improve the channel-to-channel calibration for our data.

The rms uncertainties on the final co-added spectrum of HLSW-01 range from 1.8 to $4.9 \mathrm{mJy}$, or $15 \%$ for most detectors. These errors do not include the uncertainties on the brightness temperatures of Mars and Neptune, which are $~ 5 \%$.

\section{REDSHIFT, CONTINUUM, AND LINE FLUX MEASUREMENTS}

The 190-310 GHz spectrum for HLSW-01 measured by $\mathrm{Z}$-Spec is shown as a histogram in Figure 1. The error bars represent the $1 \sigma$ photometric errors on the measurements (not including calibration errors).

\subsection{Redshift Determination}

We use a custom-developed algorithm to determine the redshift of HLSW-01 based on the detection of multiple emission lines. This algorithm is described in detail in Lupu et al. (2010, hereafter L10), and we summarize it here. Since Z-Spec (with channel widths ranging from 720 to $1290 \mathrm{~km} \mathrm{~s}^{-1}$ ) does not spectrally resolve the line emission from typical galaxies, the signal from a given line will approximately fall within a single Z-Spec channel, although the spectral response profiles of adjacent channels overlap somewhat (Earle et al. 2006). Using a reference line list containing all CO transitions up to $J=17 \rightarrow 16$ and the fine structure lines from neutral carbon ([C I $]$ ) and singly ionized carbon and nitrogen ([C II] and [N II], respectively), we compute two different estimators based on combinations of the continuum-subtracted signal-to-noise ratio $(\mathrm{S} / \mathrm{N})$ measured in all channels where the reference lines would lie for a given redshift. For HLSW-01, both estimators, $E_{1}(z)$ and $E_{2}(z)$ (see L10 


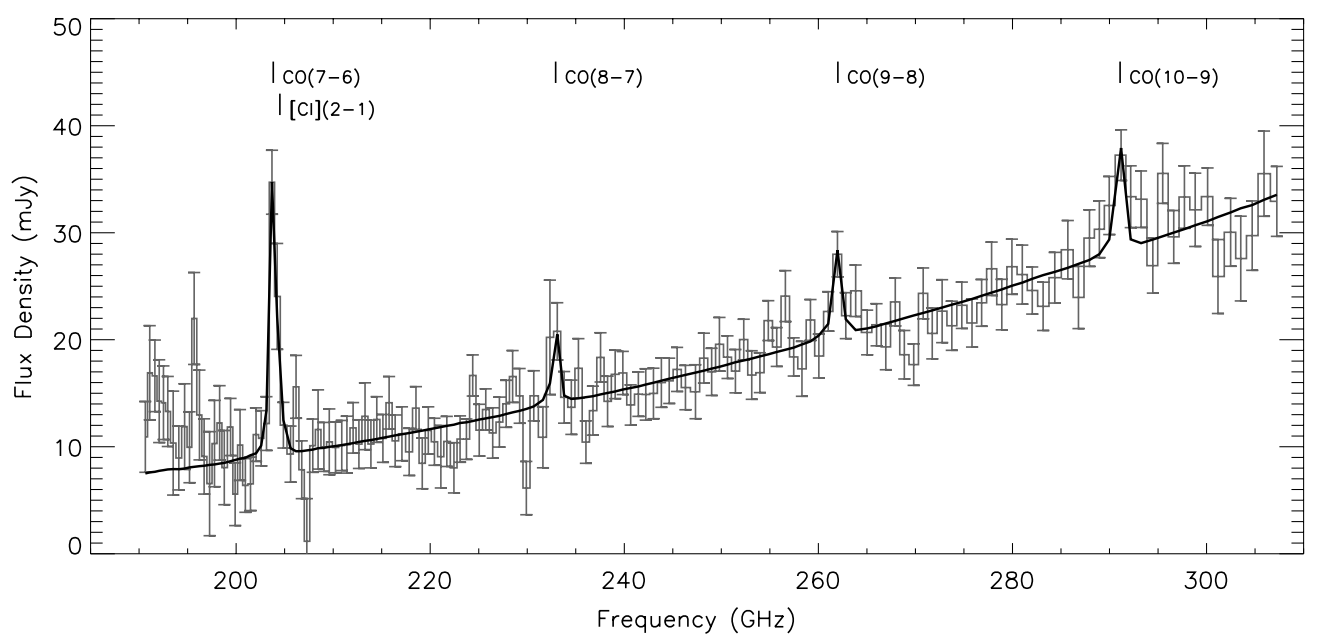

Figure 1. Millimeter wavelength spectrum for HLSW-01 measured by Z-Spec (histogram). The error bars show the $1 \sigma$ photometric errors on the measurements and do not include the 5\% uncertainty on the absolute flux calibration. The solid curve shows the best-fit model to the dust continuum and the $\mathrm{CO}$ and [C I] line emission, with the positions of the lines marked. This model includes the convolution of the intrinsic line profile $\left(\Delta v=350 \mathrm{~km} \mathrm{~s}^{-1}\right)$ with the spectral response profiles of the channels.

for details), are maximal at $z=2.958 \pm 0.007$, and with the maximum values of $E_{1}(2.958)=9.1$ and $E_{2}(2.958)=5.3$, the redshift is determined with $\gg 99.99 \%$ confidence (L10). Subsequent measurements of the $\mathrm{CO} J=5 \rightarrow 4$ line using the Institut de Radioastronomie Millimétrique (IRAM) Plateau de Bure Interferometer $(\mathrm{PdBI})$, the $\mathrm{CO} J=3 \rightarrow 2$ line using the Combined Array for Research in Millimeter Astronomy (CARMA), and the $\mathrm{CO} J=1 \rightarrow 0$ line using the GBT Zpectrometer, confirmed this redshift $(z=2.9574 \pm 0.0001$; Riechers et al. 2011, hereafter R11).

\subsection{Line and Continuum Fitting}

We fit the spectrum of HLSW-01 to a model consisting of a power-law continuum and CO line emission. Since the spectrometer outputs only a single value per channel, it is not critically sampled, and so we use the spectral response profile for each channel in the fitting. We exclude in the fit all channels with $v \leqslant 190 \mathrm{GHz}$ due to poor calibration. We fix all line widths to $350 \mathrm{~km} \mathrm{~s}^{-1}$ (full width at half-maximum), which is the line width measured by the PdBI, CARMA, and Zpectrometer assuming a Gaussian profile (R11); however, we note that the fitted line fluxes are fairly insensitive to the choice of line width. The $\mathrm{CO} J=7 \rightarrow 6$ line is separated from the $[\mathrm{CI}]{ }^{3} P_{2} \rightarrow{ }^{3} P_{1}$ (hereafter $[\mathrm{CI}] 2 \rightarrow 1$ ) fine structure line by $\sim 1000 \mathrm{~km} \mathrm{~s}^{-1}$, or roughly one Z-Spec channel. For this reason, we fix the redshift to $z=2.9574$, the value measured by the PdBI, and include the $[\mathrm{CI}] 2 \rightarrow 1$ line in the fit. The line fluxes (or $5 \sigma$ upper limits) for the four CO lines in the Z-Spec bandpass and the [CI] $2 \rightarrow 1$ line are shown in Table 1 , along with the Zpectrometer CO $J=1 \rightarrow 0$, CARMA CO $J=3 \rightarrow 2$, and PdBI CO $J=5 \rightarrow 4$ measurements (R11). We were particularly unlucky in that the $J=8 \rightarrow 7$ line falls within a noisy channel of the Z-Spec bandpass, so we obtain only an upper limit for this line. The best fit to the dust continuum is $F_{v}=(15.3 \pm 0.2)\left(\frac{v}{240 \mathrm{GHz}}\right)^{3.2 \pm 0.1} \mathrm{mJy}$. Given a dust temperature of $T_{\mathrm{d}}=88 \mathrm{~K}$ determined from fitting the FIR to mm continuum (C11), we are not strictly in the Rayleigh-Jeans limit over the entire Z-Spec bandpass at $z=2.9574$; however, this power law provides a good fit to these data (reduced $\chi^{2}$ of 0.97 ) and is sufficient for the purposes of baseline fitting. This best-fit model is overplotted in Figure 1.
The formal errors on the line fluxes derived from the above fit do not include the uncertainty of the Z-Spec frequency scale, which is $\sigma_{v} \sim 100 \mathrm{~km} \mathrm{~s}^{-1}$. This has important implications for the $\mathrm{CO} J=7 \rightarrow 6$ and $\left[\mathrm{C}_{\mathrm{I}}\right] 2 \rightarrow 1$ line fluxes, as these lines are blended. We include this additional uncertainty on the lines fluxes by shifting the redshift by $\pm \sigma_{z}= \pm(1+z) \sigma_{v} / c=$ \pm 0.001 , refitting the line fluxes, and taking the upper and lower bounds from the statistical $1 \sigma$ errors for these fits. These $\pm 1 \sigma$ uncertainties in the best-fit fluxes are listed in parentheses in Table 1. For the $\mathrm{CO} J=7 \rightarrow 6$ and [C I] $2 \rightarrow 1$ lines, including the frequency scale uncertainty roughly doubles the error bounds on the measured lines fluxes.

The CO $J=10 \rightarrow 9$ line appears somewhat broader than the other transitions and is brighter than the $J=9 \rightarrow 8$ line; we therefore consider possible blending with the $\mathrm{o}-\mathrm{H}_{2} \mathrm{O}$ $3_{12} \rightarrow 2_{21}$ water line at $v_{\text {rest }}=1153.13 \mathrm{GHz}$. Recent observations of submm-bright AGN-host galaxies show evidence for water vapor emission (Bradford et al. 2009; Lupu et al. 2010), the most striking example being the local ULIRG Mrk 231, where the Herschel SPIRE/FTS spectrum reveals seven rotational emission lines of water (González-Alfonso et al. 2010). Given the close proximity in frequency of the CO $J=10 \rightarrow 9$ and $\mathrm{o}-\mathrm{H}_{2} \mathrm{O} 3_{12} \rightarrow 2_{21}$ lines, we cannot deblend these lines within the Z-Spec spectrum; however, theoretical arguments supported by observed water line ratios in nearby galaxies suggest that the CO $J=10 \rightarrow 9$ line flux for HLSW-01 is not contaminated by water emission. The $\mathrm{o}-\mathrm{H}_{2} \mathrm{O} 3_{12} \rightarrow 2_{21}$ line is part of a de-excitation cascade process, following the excitation of the $3_{21}$ level through the o- $\mathrm{H}_{2} \mathrm{O} 2_{12} \rightarrow 3_{21} 75 \mu \mathrm{m}$ transition. The subsequent cascade results in the emission of the $\mathrm{o}-\mathrm{H}_{2} \mathrm{O} 3_{21} \rightarrow 3_{12}\left(v_{\text {rest }}=1162.91 \mathrm{GHz}\right)$, o- $\mathrm{H}_{2} \mathrm{O} 3_{12} \rightarrow 3_{03}$ $\left(v_{\text {rest }}=1097.37 \mathrm{GHz}\right.$ ), and o- $\mathrm{H}_{2} \mathrm{O} 3_{12} \rightarrow 2_{21}$ lines (e.g., see Figure 2 in González-Alfonso et al. 2010). Photon number conservation implies that the sum of the photons emitted in the $\mathrm{o}-\mathrm{H}_{2} \mathrm{O} 3_{12} \rightarrow 2_{21}$ and o- $\mathrm{H}_{2} \mathrm{O}_{12} \rightarrow 3_{03}$ lines equals the number of photons emitted in the $\mathrm{o}-\mathrm{H}_{2} \mathrm{O} 3_{21} \rightarrow 3_{12}$ line. Since neither the o- $\mathrm{H}_{2} \mathrm{O}_{21} \rightarrow 3_{12}$ nor the o- $\mathrm{H}_{2} \mathrm{O} 3_{12} \rightarrow 3_{03}$ lines are detected in the spectrum of HLSW-01 at the $1 \sigma$ level, we argue that the strength of the $\mathrm{o}-\mathrm{H}_{2} \mathrm{O} 3_{12} \rightarrow 2_{21}$ line is also consistent with the noise in our measurements. Furthermore, the observed $\mathrm{o}-\mathrm{H}_{2} \mathrm{O} 3_{21} \rightarrow 3_{12}$ and $\mathrm{p}-\mathrm{H}_{2} \mathrm{O} \quad 2_{02} \rightarrow$ $1_{11}\left(v_{\text {rest }}=987.92 \mathrm{GHz}\right)$ transitions are stronger than the 
Table 1

$\mathrm{CO}$ and $\left[\mathrm{C}_{\mathrm{I}}\right]$ Transitions Observed in HLSW-01

\begin{tabular}{|c|c|c|c|c|c|c|}
\hline Transition & $\begin{array}{c}v_{\text {rest }} \\
(\mathrm{GHz})\end{array}$ & $\begin{array}{c}v_{\mathrm{obs}} \\
(\mathrm{GHz})\end{array}$ & $\begin{array}{l}\text { Flux Density } \\
\left(\mathrm{Jy} \mathrm{km} \mathrm{s}^{-1}\right)\end{array}$ & $\begin{array}{l}\text { Line Luminosity }{ }^{\mathrm{a}, \mathrm{b}} \\
\left(10^{10} \mathrm{~K} \mathrm{~km} \mathrm{~s}^{-1} \mathrm{pc}^{2}\right)\end{array}$ & $\begin{array}{c}\text { Line Flux }{ }^{\mathrm{a}, \mathrm{b}} \\
\left(10^{-20} \mathrm{~W} \mathrm{~m}^{-2}\right)\end{array}$ & Reference \\
\hline $\mathrm{CO} J=1 \rightarrow 0$ & 115.27 & 29.13 & $1.1 \pm 0.1$ & $4.04 \pm 0.39$ & $0.010 \pm 0.001$ & GBT Zpectrometer; R11 \\
\hline $\mathrm{CO} J=3 \rightarrow 2$ & 345.80 & 87.38 & $9.7 \pm 0.5$ & $3.83 \pm 0.20$ & $0.26 \pm 0.01$ & CARMA; R11 \\
\hline $\mathrm{CO} J=5 \rightarrow 4$ & 576.27 & 145.61 & $23.6 \pm 1.4$ & $3.34 \pm 0.20$ & $1.05 \pm 0.06$ & IRAM PdBI; R11 \\
\hline $\mathrm{CO} J=7 \rightarrow 6$ & 806.65 & 203.83 & $34.6 \pm 4.2\left({ }_{-8.1}^{+9.9}\right)$ & $2.50 \pm 0.30\left(\begin{array}{c}+0.72 \\
-0.58\end{array}\right)$ & $2.2 \pm 0.3\left({ }_{-0.5}^{+0.6}\right)$ & Z-Spec; This work \\
\hline $\mathrm{CO} J=8 \rightarrow 7$ & 921.80 & 232.92 & $<20(<30)$ & $<1.1(<1.7)$ & $<1.5(<2.2)$ & Z-Spec; This work \\
\hline $\mathrm{CO} J=9 \rightarrow 8$ & 1036.91 & 262.01 & $12.7 \pm 3.3\left({ }_{-3.3}^{+3.7}\right)$ & $0.56 \pm 0.15\left({ }_{-0.14}^{+0.16}\right)$ & $1.0 \pm 0.3\left({ }_{-0.3}^{+0.3}\right)$ & Z-Spec; This work \\
\hline $\mathrm{CO} J=10 \rightarrow 9$ & 1151.99 & 291.09 & $14.7 \pm 3.7\left(\begin{array}{c}+4.9 \\
-4.1\end{array}\right)$ & $0.52 \pm 0.13\left(\begin{array}{c}+0.17 \\
-0.14\end{array}\right)$ & $1.3 \pm 0.3\left(\begin{array}{c}+0.4 \\
-0.4\end{array}\right)$ & Z-Spec; This work \\
\hline$\left[\mathrm{C}_{\mathrm{I}}\right]{ }^{3} P_{2} \rightarrow{ }^{3} P_{1}$ & 809.34 & 204.51 & $<25(<48)$ & $<1.8(<3.5)$ & $<1.6(<3.0)$ & Z-Spec; This work \\
\hline
\end{tabular}

Notes.

${ }^{a}$ For the Z-Spec data, the initial errors and $5 \sigma$ upper limits come from the formal statistical errors from fitting the spectrum. The errors and $5 \sigma$ upper limits in parentheses include the uncertainty in the Z-Spec frequency scale as described in Section 3.2.

${ }^{\mathrm{b}}$ Corrected for magnification assuming $\mu=10.9$.

o- $\mathrm{H}_{2} \mathrm{O} 33_{12} \rightarrow 2_{21}$ line for both Mrk 231 (González-Alfonso et al. 2010) and Arp 220 (N. Rangwala 2010, private communication). This adds observational evidence against significant blending of the $\mathrm{CO} J=10 \rightarrow 9$ line with the $\mathrm{o}-\mathrm{H}_{2} \mathrm{O} 3_{12} \rightarrow 2_{21}$ line in HLSW-01, since we do not detect the stronger water lines in the spectrum.

The CO SLED in units of $\mathrm{W} \mathrm{m} \mathrm{m}^{-2}$ and in line luminosity units $\left(L_{\mathrm{CO}}^{\prime}\right.$; Solomon \& Vanden Bout 2005) is shown in Figure 2. These values have been corrected for a magnification of $\mu=10.9$, as determined from the lensing model of G11. The SLED turns over at $6 \lesssim J \lesssim 8$, as is typical for SMGs and quasars (Weiß et al. 2007a, 2007b; Danielson et al. 2011); however, it is not possible to identify the turnover precisely given that the $\mathrm{CO} J=7 \rightarrow 6$ line flux may be overestimated due to blending with the $\left[\mathrm{C}_{\mathrm{I}}\right] 2 \rightarrow 1$ emission line, and we have only an upper limit on the $\mathrm{CO} J=8 \rightarrow 7$ line flux.

\section{EXCITATION AND RADIATIVE TRANSFER MODELING}

The SLED for HLSW-01 is well sampled from low- to high- $J$, and thus allows for a rigorous analysis of the CO line excitation. We use RADEX, a non-LTE radiative transfer code which uses an iterative escape probability formalism (van der Tak et al. 2007), to model the CO excitation of HLSW-01. For a specified gas density $\left(n_{\mathrm{H}_{2}}\right)$, kinetic temperature $\left(T_{\text {kin }}\right)$, and $\mathrm{CO}$ column density per unit line width $\left(N_{\mathrm{CO}} / \Delta v\right)$, RADEX calculates the excitation temperatures, line optical depths, and line surface brightnesses. For our analysis, we use the escape probability in an expanding spherical cloud and assume a $T_{\mathrm{CMB}}(z)=2.73(1+z) \mathrm{K}$ blackbody for the background radiation field. However, we note that the RADEX results are not sensitive to the precise form of escape probability chosen.

Following a similar analysis to that described in Ward et al. (2003)—see also Bradford et al. (2009), Naylor et al. (2010), and Kamenetzky et al. (2011)—we use RADEX to compute the expected CO line intensities over parameter space in $T_{\text {kin }}, n_{\mathrm{H}_{2}}$, and $N_{\mathrm{CO}}$, assuming a line width of $\Delta v=350 \mathrm{~km} \mathrm{~s}^{-1}$ (R11) for all transitions. Rather than computing line intensities over a grid in parameter space, we use a Markov Chain Monte Carlo method (Metropolis et al. 1953; Hastings 1970) to determine the likelihood distributions for these parameters by comparing the RADEX results to the observed line intensities. The integrated line fluxes, $S_{\mathrm{CO}} \Delta v$ (in Jy $\mathrm{km} \mathrm{s}^{-1}$, Table 1), are converted into Rayleigh-Jeans equivalent velocity-integrated intensities, $T_{\mathrm{R}} \Delta v$

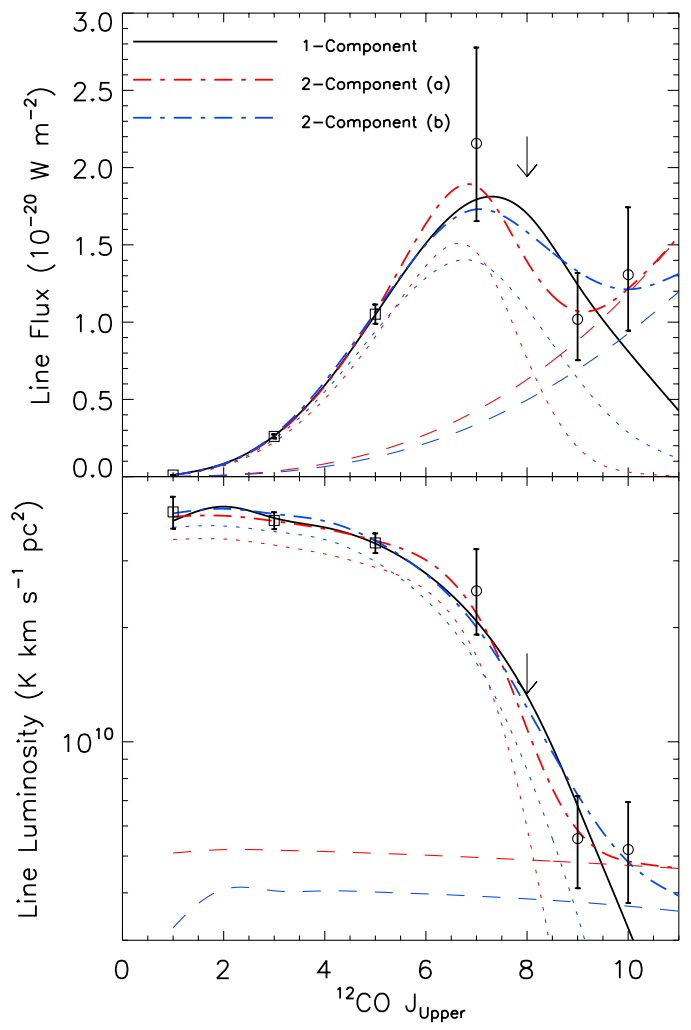

Figure 2. CO SLED for HLSW-01, in line flux (top) and line luminosity units (bottom). The circles and upper limit $(5 \sigma)$ are from the Z-Spec measurements (this work), where the $1 \sigma$ error bars include the uncertainty from the Z-Spec frequency scale. The squares are from the GBT (CO $J=1 \rightarrow 0)$, CARMA $(\mathrm{CO} J=3 \rightarrow 2$ ), and $\mathrm{PdBI}$ (CO $J=5 \rightarrow 4$ ) observations (R11). All data have been corrected for magnification assuming $\mu=10.9$. The black solid curve represents the maximum likelihood single-component model discussed in Section 5.1. The red dot-dashed curve shows the maximum likelihood solution from a two-component gas model described in Section 5.2. The red dashed and dotted curves show the contribution from the warm and cold components, respectively. The blue dot-dashed curve demonstrates a different two-component gas model (also described in Section 5.2) that provides a good fit to these data; in this case, both components (blue dashed and dotted curves) are relatively warm.

(in $\mathrm{K} \mathrm{km} \mathrm{s}^{-1}$ ) for comparison with the output from RADEX:

$$
T_{\mathrm{R}} \Delta v=\frac{c^{2}}{2 k v_{\mathrm{obs}}^{2}} \frac{S_{\mathrm{CO}} \Delta v}{\mu \Omega_{\mathrm{em}}}(1+z)
$$



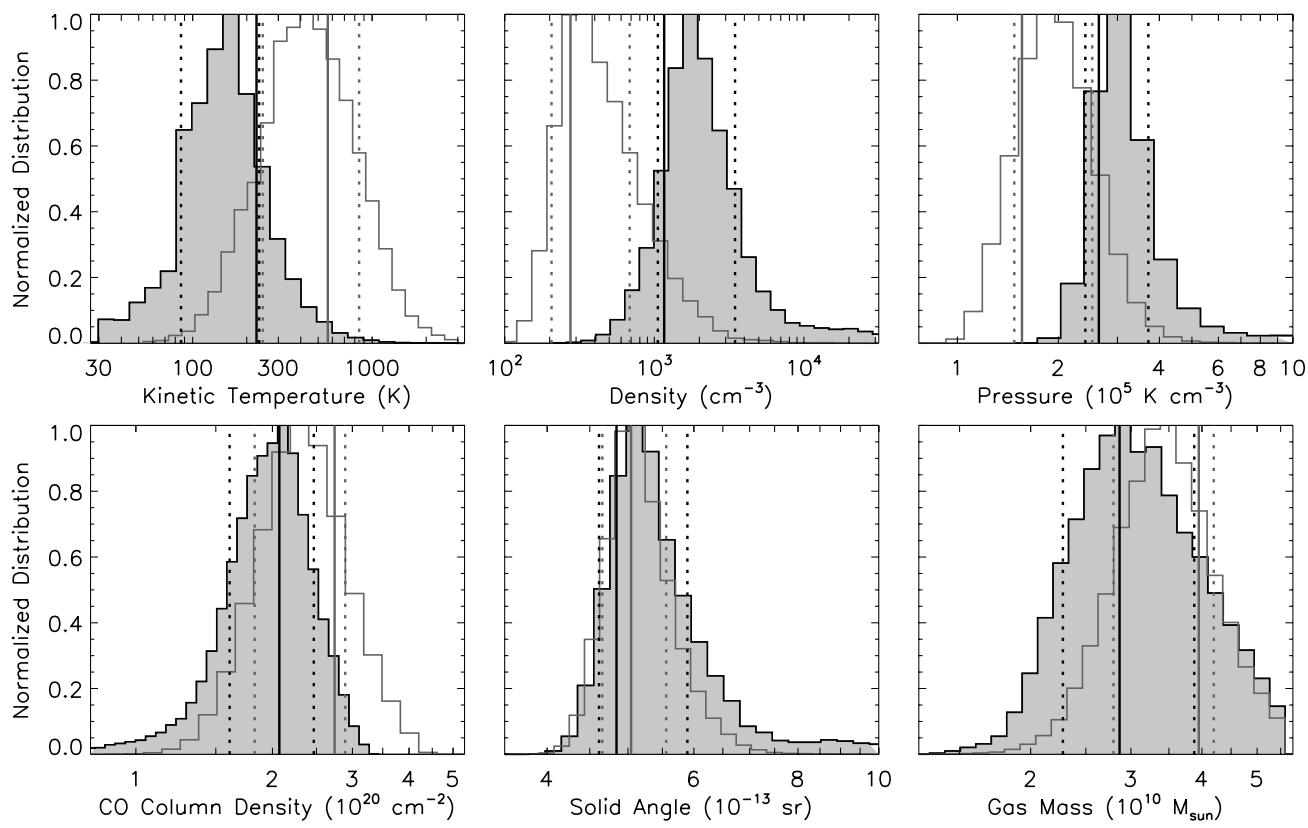

Figure 3. Top left, top center, bottom left, bottom center: marginalized distributions for the four parameters from the excitation modeling of HLSW-01. Top right, bottom right: marginalized distributions for pressure and gas mass, derived from the four primary parameters. All of the distributions are normalized at their peaks. In each panel, the gray, unshaded histogram shows the resulting distribution when the gas is not required to be self-gravitating, while the shaded histogram shows the distribution when this constraint is enforced (i.e., Equation (3)). The vertical solid and dotted lines indicate the maximum likelihood solutions and the marginalized 68.3\% confidence intervals, respectively, with (black) and without (gray) the constraint of self-gravitating gas. The source solid angle and gas mass distributions assume a gravitational lensing magnification factor of $\mu=10.9$ for HLSW-01. For a different value of $\mu$, multiply the $x$-axes in the bottom center and right plots by $\left(\frac{10.9}{\mu}\right)$.

Table 2

Results of Single-phase CO Excitation Modeling for HLSW-01

\begin{tabular}{lccc}
\hline \hline Parameter & $K_{\text {vir Unconstrained }}$ Maximum Likelihood Solution & Maximum Likelihood Solution & Units \\
\hline$T_{\text {kin }}$ & $566(246-845)$ & $227(86-235)$ & $\mathrm{K}$ \\
$n_{\mathrm{H}_{2}}$ & $0.3(0.2-0.7)$ & $1.2(1.1-3.5)$ & $10^{3} \mathrm{~cm}^{-3}$ \\
$N_{\mathrm{CO}}$ & $2.7(1.8-2.9)$ & $2.1(1.6-2.5)$ & $10^{20} \mathrm{~cm}^{-2}$ \\
$\Omega_{\mathrm{em}}$ & $5.0(4.7-5.6)$ & $4.8(4.6-5.9)$ & $\left(\frac{10.9}{\mu}\right) 10^{-13} \mathrm{sr}$ \\
\hline$P$ & $1.6(1.5-2.5)$ & $2.6(2.4-3.7)$ & $10^{5} \mathrm{~K} \mathrm{~cm}^{-3}$ \\
$M_{\text {gas }}$ & $3.9(2.8-4.2)$ & $2.9(2.3-3.9)$ & $\left(\frac{2 \times 10^{-4}}{X_{\mathrm{CO}}}\right)\left(\frac{10.9}{\mu}\right) 10^{10} M_{\odot}$ \\
$d v / d r$ & $0.22(0.18-0.55)$ & $1.2(1.1-3.0)$ & $\left(\frac{X_{\mathrm{CO}}}{2 \times 10^{-4}}\right) \mathrm{km} \mathrm{s}^{-1} \mathrm{pc}^{-1}$ \\
\hline
\end{tabular}

Notes. The columns are as follows. Column 1: the parameter; Column 2: the maximum likelihood value of the parameter and $68.3 \%$ confidence interval (in parenthesis), without the constraint that the gas is self-gravitating; Column 3: the maximum likelihood value of the parameter and $68.3 \%$ confidence interval when the gas is required to be self-gravitating (Equation (3)); and Column 4: the units for the given values.

where $\mu=10.9$ (G11) is the gravitational lensing magnification factor and $\Omega_{\mathrm{em}}$ is the intrinsic solid angle of the CO emitting region (see the next paragraph). We propagate the line flux errors using the $1 \sigma$ error bounds that include the uncertainty in the Z-Spec frequency scale. We do not include errors in the absolute flux calibration as these are small compared to the random errors on the measurements, nor do we account for systematic calibration errors among the measurements, as these are not possible to characterize without at least two measurements of the same CO transition using different telescopes.

The CO emission is only marginally resolved in both the IRAM PdBI and CARMA maps. Given the uncertainties in the size of the $\mathrm{CO}$ emitting region and the gravitational magnification, combined with the fact that the area filling factor of the molecular gas may be $<1$, we choose to include the intrinsic source solid angle, $\Omega_{\mathrm{em}}$, as a fourth parameter in our likelihood analysis. The intrinsic solid angle refers to the observed solid angle in the absence of lensing. Finally, we impose an upper limit for the kinetic temperature of $3000 \mathrm{~K}$; above this temperature, collisional dissociation of $\mathrm{CO}$ becomes important (with a weak dependence on the gas density).

We ran a 150,000 step Markov chain to determine the likelihood distributions of the gas parameters for HLSW-01. The marginalized distributions for the four parameters are shown in Figure 3 (gray, unshaded histogram), with the maximum likelihood values marked with gray solid vertical lines. Note that since these are marginalized distributions, the peaks do not necessarily coincide with the four-dimensional maximum likelihood solution. We use these one-dimensional distributions only for computing the marginalized $68.3 \%$ confidence interval on each of the parameters, which is indicated by the gray vertical dotted lines in Figure 3. These results are summarized in Table 2. Figure 4 shows the two-dimensional marginalized distributions for $T_{\text {kin }}$ and $n_{\mathrm{H}_{2}}, \Omega_{\mathrm{em}}$ and $N_{\mathrm{CO}}$, and $N_{\mathrm{CO}}$ and $n_{\mathrm{H}_{2}}$ (gray contours). 

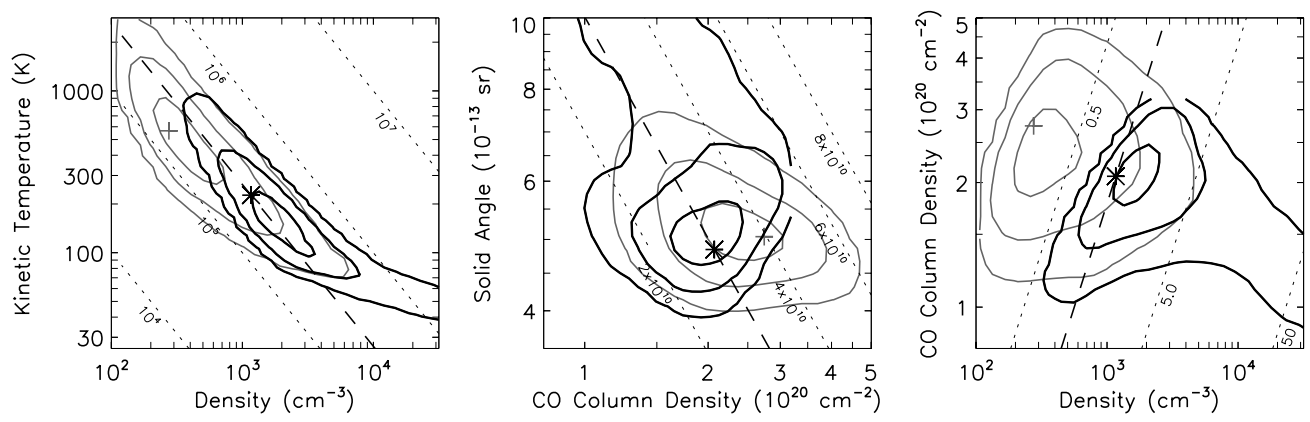

Figure 4. Two-dimensional marginalized distributions for $T_{\text {kin }}$ and $n_{\mathrm{H}_{2}}$ (left), $\Omega_{\mathrm{em}}$ and $N_{\mathrm{CO}}$ (center), and $N_{\mathrm{CO}}$ and $n_{\mathrm{H}_{2}}$ (right). In all plots, the contours are $68.3 \%$, 95.5\%, and $99.7 \%$ assuming Gaussian distributed errors. The gray contours show the resulting distributions when the gas is not constrained to be self-gravitating, and the gray plus symbols mark the maximum likelihood solution in that case. The black contours show the distributions with the constraint that the gas is self-gravitating, with the black stars marking the maximum likelihood solution. In the left plot, the dotted lines indicate lines of constant pressure (in units of $\mathrm{K} \mathrm{cm}^{-3}$ ), and the dashed pressure line passes through the maximum likelihood solution. In the center plot, the dotted lines show constant gas mass (in units of $M_{\odot}$ ), and the dashed line passes through the maximum likelihood solution. The dotted lines in the right plot show constant velocity gradient (in units of km s $\mathrm{sc}^{-1} \mathrm{pc}^{-1}$ ) where the dashed line indicates the maximum likelihood solution. The source solid angle assumes a gravitational lensing magnification of $\mu=10.9$ for HLSW-01. For a different value of $\mu$, multiply the $y$-axes in the center plot by $\left(\frac{10.9}{\mu}\right)$.

For $T_{\text {kin }}$ and $n_{\mathrm{H}_{2}}$, the contours fall along a line of constant pressure.

We also derive the gas pressure ( $\left.P=n_{\mathrm{H}_{2}} T_{\text {kin }}\right)$ and mass, where the latter is estimated as

$$
M_{\mathrm{gas}}=\frac{\Omega_{\mathrm{em}} D_{\mathrm{A}}^{2} N_{\mathrm{CO}}\left(1.4 m_{\mathrm{H}_{2}}\right)}{X_{\mathrm{CO}}},
$$

where $\Omega_{\mathrm{em}} D_{\mathrm{A}}^{2}$ is the area of the emitting region, $D_{\mathrm{A}}$ is the angular diameter distance, $X_{\mathrm{CO}}=n_{\mathrm{CO}} / n_{\mathrm{H}_{2}}$ is the relative abundance of $\mathrm{CO}$ to $\mathrm{H}_{2}$, and $m_{\mathrm{H}_{2}}$ is the mass of the hydrogen molecule (with a factor of 1.4 to account for helium). We assume $X_{\mathrm{CO}}=2 \times 10^{-4}$, a reasonable value based on observations of nearby galaxies and star-forming regions in the Galaxy (e.g., Wang et al. 2004). The marginalized distributions for these secondary parameters are also shown in Figure 3.

The maximum likelihood solution gives $T_{\text {kin }} \approx 570 \mathrm{~K}$ and $n_{\mathrm{H}_{2}} \approx 3 \times 10^{2} \mathrm{~cm}^{-3}$, a condition that is unlike the average molecular gas seen in most galaxies. This solution is also inconsistent with a gas that has at least enough velocity dispersion to correspond to virialized motion under its own self-gravity. Using the notation of Papadopoulos et al. (2007) and reforming their Equation (2) for our parameterization yields the following constraint for a self-gravitating gas:

$$
K_{\mathrm{vir}} \equiv \frac{(d v / d r)_{\mathrm{obs}}}{(d v / d r)_{\mathrm{vir}}}=\frac{\Delta v n_{\mathrm{H}_{2}}^{1 / 2} X_{\mathrm{CO}}}{\left(4 \pi G \alpha\left(1.4 m_{\mathrm{H}_{2}}\right) / 3\right)^{1 / 2} N_{\mathrm{CO}}} \geqslant 1,
$$

where $\alpha=1-2.5$ depending on the cloud density profile, and we estimate the velocity gradient for an individual cloud as $(d v / d r)_{\mathrm{obs}}=\left(\frac{\Delta v}{N_{\mathrm{CO}}}\right) n_{\mathrm{H}_{2}} X_{\mathrm{CO}}$ (Kamenetzky et al. 2011). Assuming $X_{\mathrm{CO}}=2 \times 10^{-4}, K_{\mathrm{vir}}=0.2-0.4$ for the maximum likelihood solution. For this reason, we add a prior to our likelihood analysis that constrains parameter space to solutions where the gas is self-gravitating, i.e., where Equation (3) holds, and we repeat our Markov chain calculations. These results are presented in Table 2, Figure 3 (shaded histograms), and Figure 4 (black contours), where the maximum likelihood solution now gives $T_{\text {kin }} \approx 230 \mathrm{~K}$ and $n_{\mathrm{H}_{2}} \approx 1.2 \times 10^{3} \mathrm{~cm}^{-3}$. Throughout the rest of this paper, we discuss only the solution where the constraint on $K_{\text {vir }}$ has been enforced.

\section{RESULTS AND DISCUSSION}

Our likelihood analysis for HLSW-01 suggests that the $\mathrm{CO}$ gas is primarily tracing warm, moderate-density gas with
$T_{\text {kin }}=227_{-141}^{+8} \mathrm{~K}$ and $n_{\mathrm{H}_{2}}=1.2_{-0.1}^{+2.3} \times 10^{3} \mathrm{~cm}^{-3}$. This singlecomponent model provides a good fit to our data, agreeing within $1.5 \sigma$ of all measurements as demonstrated in Figure 2, where the model line fluxes corresponding to our maximum likelihood solution (solid black curve) are compared to our data. However, this model falls short of the $J=10 \rightarrow 9$ line flux, and it is likely that the full CO SLED is really a composite of multiple gas components, as seen in many nearby starbursts and highredshift galaxies (Ward et al. 2003; Weiß et al. 2007a; Greve et al. 2009; Panuzzo et al. 2010; Loenen et al. 2010; Riechers et al. 2010; Danielson et al. 2011; Carilli et al. 2010; van der Werf et al. 2010). In Section 5.1, we discuss the implications our single-component gas model would have for HLSW-01, and in Section 5.2, we consider a few two-phase gas models that are also consistent with our data.

\subsection{Single-component Gas Model}

For our best-fit single-component gas model, all of the CO transitions are sub-thermally populated, with an excitation temperature of $T_{\mathrm{ex}} \sim 135 \mathrm{~K}$ for the $\mathrm{CO} J=1 \rightarrow 0$ line, and decreasing to $T_{\mathrm{ex}} \sim 25 \mathrm{~K}$ for the $\mathrm{CO} J=10 \rightarrow 9$ line. The high gas kinetic temperature $\left(T_{\text {kin }}=230 \mathrm{~K}\right)$ is required to excite the higher- $J$ transitions, and is considerably larger than the dust temperature of $T_{\mathrm{d}}=88 \mathrm{~K}$ determined from $\mathrm{C} 11$. This suggests that heating mechanisms that effectively transfer energy into the gas (e.g., X-ray-dominated regions, or heating by the dissipation of turbulence or cosmic rays) may be present in this system (although colder gas solutions that match the dust temperature are also consistent with our data).

Our result for HLSW-01 is similar to that for the local starburst galaxy M82, where the $J_{\text {up }} \geqslant 4$ lines are best fit by warm gas with moderate density $\left(T_{\text {kin }} \approx 550 \mathrm{~K}, n_{\mathrm{H}_{2}} \approx 5 \times 10^{3} \mathrm{~cm}^{-3}\right.$; Panuzzo et al. 2010). However, in contrast to M82, this warm gas is able to account for the excitation of the lower- $J$ lines as well, whereas in M82 the $J_{\text {up }} \leqslant 3$ line fluxes are dominated by a cold, less dense component (Wild et al. 1992; Guesten et al. 1993; Mao et al. 2000; Ward et al. 2003; Weiß et al. 2005b). Given its low critical density, the $\mathrm{CO} J=1 \rightarrow 0$ line is easily excited in both cold, diffuse gas as well as warm, dense gas in star-forming regions. There is growing evidence that many high-redshift SMGs contain a significant amount ( $\sim 50 \%$ of the total mass) of extended, cold gas that is not associated with the nuclear starburst (e.g., Ivison et al. 2010a; Danielson et al. 2011; Carilli et al. 2010). This leads to an excess in the CO 
$J=1 \rightarrow 0$ line luminosity relative to the higher- $J$ transitions, with a line ratio of $R_{3,1} \equiv L_{\mathrm{CO}(3-2)}^{\prime} / L_{\mathrm{CO}(1-0)}^{\prime} \sim 0.6$ (e.g., Harris et al. 2010; Frayer et al. 2011; Ivison et al. 2010a) and a line width ratio of $\Delta v_{\mathrm{CO}(1-0)} / \Delta v_{\mathrm{CO}(3-2)}=1.15 \pm 0.06$ (Ivison et al. 2010a). However, this does not appear to be the case for HLSW-01, where $R_{3,1}=0.95$ and the observed line widths for the $J_{\text {up }}=1,3$, and 5 transitions are all consistent with the presence of a single component. This suggests that the $J_{\text {up }} \leqslant 5$ transitions for HLSW-01 are largely tracing the same volume of molecular gas in this galaxy, and given the large uncertainties in the higher- $J$ observations, we see no strong evidence that a two-phase gas model is needed to explain the $\mathrm{CO}$ excitation for this source.

We derive a source solid angle of $\Omega_{\mathrm{em}}=4.8_{-0.2}^{+1.1}\left(\frac{10.9}{\mu}\right) \times$ $10^{-13} \mathrm{sr}$, which corresponds to an equivalent diameter of $1.25_{-0.03}^{+0.14}\left(\frac{10.9}{\mu}\right)^{1 / 2} \mathrm{kpc}$ at $z=2.9574$, assuming spherical geometry. Combined with the warm temperature and modest density, this suggests that the molecular gas is excited by an intense starburst that is less centrally concentrated than that seen in local ULIRGs, but consistent with previous findings of extended star formation in SMGs (1.5-3 kpc; e.g., Biggs \& Ivison 2008; Tacconi et al. 2008; Lestrade et al. 2010; Swinbank et al. 2010). It is possible that the molecular gas reservoir is extended over an even larger area (e.g., Ivison et al. 2010a; Carilli et al. 2010), and $\Omega_{\mathrm{em}}$ really represents the product of this area with a filling fraction. The velocity gradients observed for the four images in the $\mathrm{CO} J=3 \rightarrow 2$ and $\mathrm{CO} J=5 \rightarrow 4$ maps suggest a complex velocity structure, possibly arising from a major merger (R11); however, higher spatial resolution CO maps are needed in order to confirm this interpretation.

The total molecular gas mass derived from this line excitation modeling (Equation (2)) is $2.9_{-0.6}^{+1.0}\left(\frac{2 \times 10^{-4}}{X_{\mathrm{CO}}}\right)\left(\frac{10.9}{\mu}\right) \times 10^{10} M_{\odot}$, similar to those of other high-redshift SMGs and quasars (e.g., Tacconi et al. 2008; Coppin et al. 2008; Wang et al. 2010; Riechers et al. 2010). This value is consistent with the estimate from $L_{\mathrm{CO}(1-0)}^{\prime}\left(3.3 \times 10^{10} M_{\odot} ; \mathrm{R} 11\right)$ assuming a conversion factor of $\alpha_{\mathrm{CO}}=0.8 M_{\odot}\left(\mathrm{K} \mathrm{km} \mathrm{s}^{-1} \mathrm{pc}^{2}\right)^{-1}$, a value appropriate for ultra-luminous infrared galaxies (Downes \& Solomon 1998; Solomon \& Vanden Bout 2005). C11 derive an SFR of $2460 \pm 160 M_{\odot} \mathrm{yr}^{-1}$ (corrected for lensing) from the FIR luminosity (Kennicutt 1998). Assuming that all of the molecular gas will be consumed by star formation, this implies a gas depletion timescale of $11.7_{-2.5}^{+4.2} \mathrm{Myr}$ - a value similar to (12 Myr; Ivison et al. 2010a) or somewhat shorter than ( 40 Myr; Greve et al. 2005; Solomon \& Vanden Bout 2005) the mean gas depletion timescale of typical SMGs with similar luminosities. However, it is possible that the SFR is overestimated if a significant fraction of the dust is heated by AGN activity, and this estimate also neglects feedback mechanisms.

At the resolution of the existing interferometry measurements, the SMA $880 \mu \mathrm{m}$ and CO $J=3 \rightarrow 2$ and $J=5 \rightarrow 4$ maps show consistent source sizes, suggesting that the dust and gas are well mixed and therefore trace the same regions. We estimate the dust mass from the optical depth and the dust absorption coefficient, where

$$
M_{\mathrm{dust}}=\Omega_{\mathrm{em}} D_{\mathrm{A}}^{2} \frac{\tau_{v}}{\kappa_{v}},
$$

$\kappa_{v}=2.64 \mathrm{~m}^{2} \mathrm{~kg}^{-1}(v / 2400 \mathrm{GHz})^{\beta}$ (Dunne et al. 2003), and $\tau_{v}=\left(v / v_{0}\right)^{\beta}$, with $\beta=1.94 \pm 0.14$ and $v_{0}=1550 \pm 150 \mathrm{GHz}$ determined from fitting a modified blackbody to the full FIR to mm dust SED of HLSW-01 (C11). We derive a dust mass of $M_{\mathrm{d}}=5.2_{-1.1}^{+1.6} \times 10^{8} M_{\odot}$ (not including errors on $\kappa_{v}$ ), and a dust to gas mass ratio of $\lesssim 1 / 55$, considering that the total gas mass could be larger since we only estimate the mass of the molecular gas. This is consistent with the dust to gas mass ratios of typical SMGs ( 1/60; e.g., Kovács et al. 2006; Coppin et al. 2008; Michałowski et al. 2010; Santini et al. 2010). Our dust mass estimate is $\sim 5$ times higher than that estimated via $L_{\text {FIR }}$ from the single-component dust model of $\mathrm{C} 11$, assuming the same $\kappa_{v}$. This suggests that (1) a single-component model for the dust (and/or CO) emission is not a good representation of the physical properties of this galaxy, and/or (2) the gas and dust are not co-spatial. Longer wavelength continuum measurements are needed to detect the presence of multi-component dust, while higher spatial resolution imaging of the dust and $\mathrm{CO}$ would allow us to determine their relative spatial distribution.

Recent results by Papadopoulos et al. (2010b) suggest that for many (U)LIRGs, including Arp 220 (Papadopoulos et al. 2010a), high dust extinction can suppress the high-J CO line fluxes. Assuming a single-component dust model for HLSW-01 (C11), the dust optical depths at the CO $J_{\text {up }}=7-10$ rest frequencies are $\tau_{v} \sim 0.3-0.6$, and thus may be non-negligible. However, we cannot definitively know whether this effect is important for HLSW-01 given the degeneracy between high dust optical depths and large amounts of cold dust. A two-temperature component fit to the dust SED is poorly constrained and does not represent an improvement over a single-component model (C11). However, this fit only includes rest-frame frequencies $v \gtrsim 900 \mathrm{GHz}$, and it is possible that a significant amount of cold dust which is best traced at lower frequencies (Dunne et al. 2000; Dunne \& Eales 2001) is present in this galaxy. Furthermore, to properly include this effect in the excitation modeling would require knowledge of the relative geometry of the molecular gas and dust, which cannot be deduced from our current data. High-resolution mapping of the molecular gas and dust emission may shed light on this issue in the future.

\subsection{Two-component Gas Models}

The single-component gas model discussed in the previous section provides a good fit to the CO SLED for HLSW-01. However, in light of recent results showing that the $\mathrm{CO}$ excitation for a large number of local starbursts and high-redshift SMGs requires multiple gas phases, we explore a few twocomponent gas models in this section. With only six line fluxes measured, we cannot well constrain a full eight-parameter fit; however, we can place simple constraints on some of the parameters in order to test solutions that resemble those of other high-redshift SMGs.

We explore the possibility that the $\mathrm{CO}$ excitation for HLSW-01 arises from the combination of a dense, star-forming component and cold, lower density gas that is not necessarily associated with star formation. We require that the star-forming component has a gas density of $n_{\mathrm{H}_{2}}=10^{3.5}-10^{6} \mathrm{~cm}^{-3}$ and a kinetic temperature of $T_{\text {kin }} \geqslant 30 \mathrm{~K}$, which is typical of starforming regions in starburst nuclei (e.g., Riechers et al. 2010; Carilli et al. 2010; Danielson et al. 2011). We then require that the other component has a lower density and temperature than the star-forming component, but a larger emitting region (or alternatively, filling fraction). We use the same prior on the velocity gradient described in Section 4 for the star-forming component, but we relax the prior on the velocity gradient of the second component so that it is not required to be self-gravitating. The two-dimensional marginalized distributions for $T_{\text {kin }}$ and $n_{\mathrm{H}_{2}}$ for each component are shown in Figure 5. 


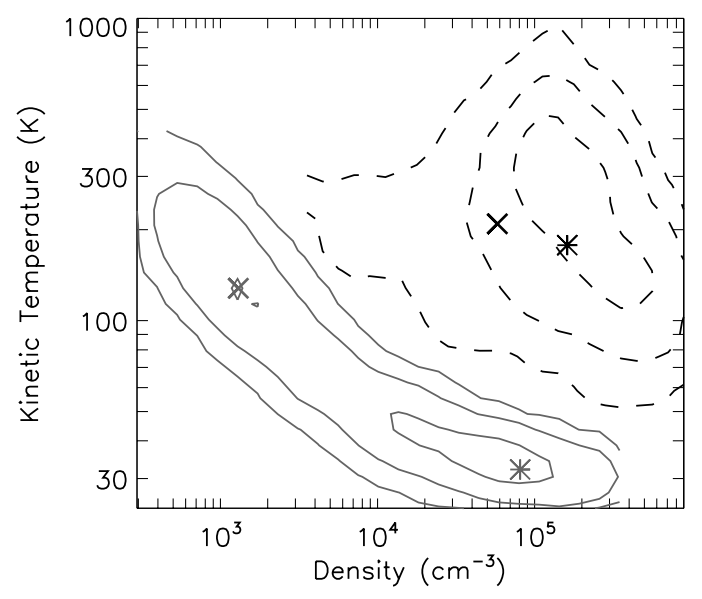

Figure 5. Two-dimensional marginalized distributions for $T_{\mathrm{kin}}$ and $n_{\mathrm{H}_{2}}$ for each component in the two-phase gas model described in Section 5.2. The contours are $68.3 \%, 95.5 \%$, and $99.7 \%$ assuming Gaussian distributed errors, and the stars mark the maximum likelihood solution (model "two-component (a)" in Figure 2). The crosses mark another solution that provides a good fit to the data (model "two-component (b)" in Figure 2).

The maximum likelihood solution for this two-component model under the above constraints is $T_{\text {kin }}=180 \mathrm{~K}, n_{\mathrm{H}_{2}}=$ $1.6 \times 10^{5} \mathrm{~cm}^{-3}$ for the star-forming component, and $T_{\text {kin }}=$ $30 \mathrm{~K}, n_{\mathrm{H}_{2}}=8.1 \times 10^{4} \mathrm{~cm}^{-3}$ for the other component. This model is shown in Figure 2 (model "two-component (a)"), where the dashed red curve corresponds to the star-forming component, the dotted red curve corresponds to the cooler component, and the dot-dashed red curve shows the summed SLED from both components. The best-fit model thus consists of two dense gas phases, with warm gas distributed over a small (330 pc equivalent diameter) region (assuming a filling fraction of one), and cold gas distributed over a larger ( $2.4 \mathrm{kpc}$ diameter) region. The total molecular gas mass is $5.1 \times 10^{10} M_{\odot}$, with $30 \%$ and $70 \%$ of the gas mass in the warm and cold phases, respectively. The warm, dense component remains thermalized out to $J_{\text {up }}=10$, and provides the best match to our $J_{\text {up }}=9-10$ measurements, where these lines have roughly equal brightness temperatures.

However, the likelihood space for this two-component model does not have a single well-defined peak, and many other solutions reproduce the lines fluxes nearly as well. For example, a warm/dense component with $T_{\text {kin }}=210 \mathrm{~K}, n_{\mathrm{H}_{2}}=$ $5.8 \times 10^{4} \mathrm{~cm}^{-3}$, and a warm/moderate-density component with $T_{\text {kin }}=130 \mathrm{~K}, n_{\mathrm{H}_{2}}=1.3 \times 10^{3} \mathrm{~cm}^{-3}$ provides a good fit to the data as well. This model is shown by the blue curves in Figure 2 (model "two-component (b)"), where the dashed, dotted, and dot-dashed curves show the warm/dense and warm/ moderate-density components, and their sum, respectively. In this scenario, the warm/dense gas is concentrated in a region $\sim 270 \mathrm{pc}$, while the warm/moderate-density gas is extended over a larger region $(\sim 1.4 \mathrm{kpc})$. The total molecular gas mass is $4.3 \times 10^{10} M_{\odot}$, with $15 \%$ and $85 \%$ of the gas mass in the warm/dense and warm/moderate-density component, respectively. We note that this latter component is similar to the results from our one-component fit, where the addition of a small fraction of warm/dense gas brings the $J=10 \rightarrow 9$ model flux in better agreement with the data.

Our data show no evidence for two-component models with a significant contribution from extended, cold, moderate-density gas with $T_{\text {kin }} \sim 30-50 \mathrm{~K}$ and $n_{\mathrm{H}_{2}} \sim 10^{2}-10^{3.5} \mathrm{~cm}^{-3}$ like that seen in M82 (Panuzzo et al. 2010) and many high-redshift galaxies (e.g., Riechers et al. 2010; Danielson et al. 2011; Carilli et al. 2010), as can be seen in Figure 5. While recent results by Harris et al. (2010) and Ivison et al. (2010a) suggest that most SMGs contain a large fraction ( $\gtrsim 50 \%$ of the total mass) of extended, cold gas with moderate-density, HLSW-01 appears to be an exception.

\subsection{Excitation Mechanisms}

The CO measurements for HLSW-01 do not have the statistical power to discriminate between various mechanisms for heating the molecular gas, such as UV radiation from star formation in photon-dominated regions (PDRs) or X-rays from an AGN in an X-ray-dominated region (XDR). The $J_{\text {up }} \geqslant 7$ line fluxes measured with Z-Spec have large uncertainties, and it is these high- $J$ transitions that can most strongly discriminate between such heating mechanisms. While high gas temperatures like that found for HLSW-01 (for both the single- and two-component models) are usually associated with heating in XDRs for sources that are known to host powerful AGNs (Weiß et al. 2007a; Ao et al. 2008), heating by cosmic rays or dissipation of turbulence can also boost the high- $J$ line fluxes; these latter mechanisms are thought to be responsible for the high excitation for M82, which does not host a strong AGN (Panuzzo et al. 2010). Based on the high dust temperature and $1.4 \mathrm{GHz}$ radio emission (in excess of that expected from the FIR-radio relation; C11), there is tentative evidence for a bolometrically important AGN in HLSW-01.

Obtaining higher $\mathrm{S} / \mathrm{N}$ measurements of the $J_{\text {up }}=7-10$ transitions-which are all accessible from ground-based telescopes-and spectrally resolving these lines would go a long way to help differentiate between various one- and two-phase gas models, and to better constrain the heating mechanism for the gas. In particular, high spectral resolution is needed to properly deblend the $\mathrm{CO} J=7 \rightarrow 6$ line from the [CI] $2 \rightarrow 1$ line. Directly measuring the line widths for these high- $J$ transitions can also help to determine whether they are tracing the same regions as the low- $J$ lines.

\section{CONCLUSIONS}

We have measured a $\mathrm{CO}$ redshift of $z=2.958 \pm 0.007$ for the multiply imaged, lensed galaxy HLSW-01 using Z-Spec on the CSO. Four rotational transitions of CO lie within the Z-Spec bandpass $\left(J_{\text {up }}=7-10\right)$ at this redshift, and we have used a well tested algorithm (L10) to robustly identify the redshift for this source with $\gg 99.99 \%$ confidence. This redshift has been confirmed with subsequent observations with the IRAM PdBI, CARMA, and the GBT Zpectrometer.

With six CO line measurements and one upper limit, the SLED for HLSW-01 is well sampled from low- to high- $J$, and we carry out a likelihood analysis using predictions from RADEX to constrain the molecular gas properties for this galaxy. We find that a single-component, warm/moderate-density gas with $T_{\text {kin }}=86-235 \mathrm{~K}$ and $n_{\mathrm{H}_{2}}=(1.1-3.5) \times 10^{3} \mathrm{~cm}^{-3}$ provides a good fit to these data. However, several two-component gas models can describe the CO SLED as well; higher S/N observations of the high- $J$ lines with finer spectral resolution are needed in order to differentiate between these scenarios.

Based on the nearly equal line luminosities measured for the $J=1 \rightarrow 0$, and $J=3 \rightarrow 2$ transitions, these data are inconsistent with models that require an extended, cold/moderate-density gas component. This is in contrast to recent results that show that the $\mathrm{CO} J=1 \rightarrow 0$ line flux for most high-redshift SMGs, as well as nearby starburst galaxies 
such as M82, requires the existence of such a low-excitation component. High spatial resolution $\mathrm{CO}$ maps of both low- and high-J lines with CARMA, the IRAM PdBI, and the EVLA could potentially provide more concrete information on the presence and distribution of cold and warm molecular gas within this galaxy.

We thank the anonymous referees for their suggestions, which improved the presentation of these results. We also thank the staff at CSO for support of the Z-Spec observations, and we appreciate the help of Robert Hanni and Jon Rodriguez with observing. J.E.A. acknowledges support by NSF grant AST-0807990 and by the CSO NSF Cooperative Agreement AST-0838261. J.K. acknowledges funding through an NSF graduate research fellowship. A.J.B. acknowledges support from NSF grant AST-0708653. Z-spec was constructed under NASA SARA grants NAGS-11911 and NAGS-12788 and an NSF Career grant (AST-0239270) and a Research Corporation Award (RI0928) to J.G., in collaboration with the Jet Propulsion Laboratory, California Institute of Technology, under a contract with the National Aeronautics and Space Administration. We acknowledge Peter Ade and his group for their filters and Lionel Duband for the $3 \mathrm{He} / 4 \mathrm{He}$ refrigerator in $\mathrm{Z}-\mathrm{Spec}$, and are grateful for their help in the early integration of the instrument.

Facilities: CSO (Z-Spec), GBT (Zpectrometer), IRAM: Interferometer (PdBI), CARMA, SMA, Herschel (SPIRE)

\section{REFERENCES}

Ao, Y., Weiß, A., Downes, D., Walter, F., Henkel, C., \& Menten, K. M. 2008, A\&A, 491, 747

Aravena, M., et al. 2010, ApJ, 718, 177

Aretxaga, I., et al. 2007, MNRAS, 379, 1571

Austermann, J. E., et al. 2010, MNRAS, 401, 160

Bertoldi, F., et al. 2007, ApJS, 172, 132

Biggs, A. D., \& Ivison, R. J. 2008, MNRAS, 385, 893

Biggs, A. D., et al. 2010, arXiv:1012.0305

Blain, A. W., Smail, I., Ivison, R. J., Kneib, J.-P., \& Frayer, D. T. 2002, Phys. Rep., 369, 111

Bothwell, M. S., et al. 2010, MNRAS, 405, 219

Bradford, C. M., et al. 2009, ApJ, 705, 112

Carilli, C. L., et al. 2010, ApJ, 714, 1407

Chapman, S. C., Blain, A. W., Smail, I., \& Ivison, R. J. 2005, ApJ, 622, 772

Chapman, S. C., et al. 2008, ApJ, 689, 889

Conley, A., et al. 2011, ApJ, submitted

Coppin, K., et al. 2006, MNRAS, 372, 1621

Coppin, K. E. K., et al. 2008, MNRAS, 389, 45

Danielson, A. L. R., et al. 2011, MNRAS, 410, 1687

Downes, D., \& Solomon, P. M. 1998, ApJ, 507, 615

Downes, D., \& Solomon, P. M. 2003, ApJ, 582, 37

Dunne, L., \& Eales, S. A. 2001, MNRAS, 327, 697

Dunne, L., Eales, S. A., \& Edmunds, M. G. 2003, MNRAS, 341, 589

Dunne, L., Eales, S., Edmunds, M., Ivison, R., Alexander, P., \& Clements, D. L. 2000, MNRAS, 315, 115

Eales, S., et al. 2010, PASP, 122, 499

Earle, L., et al. 2006, Proc. SPIE, 6275, 627510

Engel, H., et al. 2010, ApJ, 724, 233

Frayer, D. T., Ivison, R. J., Scoville, N. Z., Yun, M., Evans, A. S., Smail, I., Blain, A. W., \& Kneib, J. 1998, ApJ, 506, L7

Frayer, D. T., et al. 1999, ApJ, 514, L13

Frayer, D. T., et al. 2008, ApJ, 680, L21

Frayer, D. T., et al. 2011, ApJ, 726, L22

Gavazzi, R., et al. 2011, ApJ, submitted

Genzel, R., Baker, A. J., Tacconi, L. J., Lutz, D., Cox, P., Guilloteau, S., \& Omont, A. 2003, ApJ, 584, 633

González-Alfonso, E., et al. 2010, A\&A, 518, L43

Greve, T. R., Ivison, R. J., Bertoldi, F., Stevens, J. A., Dunlop, J. S., Lutz, D., \& Carilli, C. L. 2004, MNRAS, 354, 779

Greve, T. R., Papadopoulos, P. P., Gao, Y., \& Radford, S. J. E. 2009, ApJ, 692, 1432
Greve, T. R., et al. 2005, MNRAS, 359, 1165

Griffin, M. J., et al. 2010, A\&A, 518, L3

Guesten, R., Serabyn, E., Kasemann, C., Schinckel, A., Schneider, G., Schulz, A., \& Young, K. 1993, ApJ, 402, 537

Harris, A. I., Baker, A. J., Zonak, S. G., Sharon, C. E., Genzel, R., Rauch, K., Watts, G., \& Creager, R. 2010, ApJ, 723, 1139

Hastings, W. K. 1970, Biometrika, 57, 97

Ikarashi, S., et al. 2010, arXiv:1009.1455

Ivison, R. J., Papadopoulos, P. P., Smail, I., Greve, T. R., Thomson, A. P., Xilouris, E. M., \& Chapman, S. C. 2010a, arXiv:1009.0749

Ivison, R. J., Smail, I., Frayer, D. T., Kneib, J., \& Blain, A. W. 2001, ApJ, 561, L45

Ivison, R. J., et al. 2010b, A\&A, 518, L35

Kamenetzky, J., et al. 2011, ApJ, in press (arXiv:1102.3915)

Kennicutt, R. C., Jr. 1998, ARA\&A, 36, 189

Kneib, J.-P., Neri, R., Smail, I., Blain, A., Sheth, K., van der Werf, P., \& Knudsen, K. K. 2005, A\&A, 434, 819

Kovács, A., Chapman, S. C., Dowell, C. D., Blain, A. W., Ivison, R. J., Smail, I., \& Phillips, T. G. 2006, ApJ, 650, 592

Lestrade, J., Combes, F., Salomé, P., Omont, A., Bertoldi, F., André, P., \& Schneider, N. 2010, A\&A, 522, L4

Loenen, A. F., et al. 2010, A\&A, 521, L2

Lupu, R. E., et al. 2010, arXiv:1009.5983

Mao, R. Q., Henkel, C., Schulz, A., Zielinsky, M., Mauersberger, R., Störzer, H., Wilson, T. L., \& Gensheimer, P. 2000, A\&A, 358, 433

Metropolis, N., Rosenbluth, A. W., Rosenbluth, M. N., Teller, A. H., \& Teller, E. 1953, J. Chem. Phys., 21, 1087

Michałowski, M. J., Watson, D., \& Hjorth, J. 2010, ApJ, 712, 942

Naylor, B. J., et al. 2003, Proc. SPIE, 4855, 239

Naylor, B. J., et al. 2010, ApJ, 722, 668

Negrello, M., et al. 2010, Science, 330, 800

Neri, R., et al. 2003, ApJ, 597, L113

Oliver, S. J., et al. 2010, A\&A, 518, L21

Panuzzo, P., et al. 2010, A\&A, 518, L37

Papadopoulos, P. P., Isaak, K. G., \& van der Werf, P. P. 2007, ApJ, 668, 815

Papadopoulos, P. P., Isaak, K., \& van der Werf, P. 2010a, ApJ, 711, 757

Papadopoulos, P. P., van der Werf, P., Isaak, K., \& Xilouris, E. M. 2010b, ApJ, 715,775

Perera, T. A., et al. 2008, MNRAS, 391, 1227

Pilbratt, G. L., et al. 2010, A\&A, 518, L1

Pope, A., et al. 2006, MNRAS, 370, 1185

Riechers, D. A., et al. 2006, ApJ, 650, 604

Riechers, D. A., et al. 2010, ApJ, 720, L131

Riechers, D., et al. 2011, ApJL, submitted

Santini, P., et al. 2010, A\&A, 518, L154

Scott, K. S., et al. 2010, MNRAS, 405, 2260

Scott, S. E., et al. 2002, MNRAS, 331, 817

Sheth, K., Blain, A. W., Kneib, J.-P., Frayer, D. T., van der Werf, P. P., \& Knudsen, K. K. 2004, ApJ, 614, L5

Solomon, P. M., \& Vanden Bout, P. A. 2005, ARA\&A, 43, 677

Swinbank, A. M., et al. 2010, Nature, 464, 733

Tacconi, L. J., et al. 2006, ApJ, 640, 228

Tacconi, L. J., et al. 2008, ApJ, 680, 246

van der Tak, F. F. S., Black, J. H., Schöier, F. L., Jansen, D. J., \& van Dishoeck, E. F. 2007, A\&A, 468, 627

van der Werf, P. P., et al. 2010, A\&A, 518, L42

Vieira, J. D., et al. 2010, ApJ, 719, 763

Wang, M., Henkel, C., Chin, Y., Whiteoak, J. B., Hunt Cunningham, M., Mauersberger, R., \& Muders, D. 2004, A\&A, 422, 883

Wang, R., et al. 2010, ApJ, 714, 699

Ward, J. S., Zmuidzinas, J., Harris, A. I., \& Isaak, K. G. 2003, ApJ, 587, 171

Weiß, A., Downes, D., Neri, R., Walter, F., Henkel, C., Wilner, D. J., Wagg, J., \& Wiklind, T. 2007a, A\&A, 467, 955

Weiß, A., Downes, D., Walter, F., \& Henkel, C. 2005a, A\&A, 440, L45

Weiß, A., Downes, D., Walter, F., \& Henkel, C. 2007b, in ASP Conf. Ser. 375, From Z-Machines to ALMA: (Sub)Millimeter Spectroscopy of Galaxies, ed. A. J. Baker et al. (San Francisco, CA: ASP), 25

Weiß, A., Ivison, R. J., Downes, D., Walter, F., Cirasuolo, M., \& Menten, K. M. 2009a, ApJ, 705, L45

Weiß, A., Walter, F., \& Scoville, N. Z. 2005b, A\&A, 438, 533

Weiß, A., et al. 2009b, ApJ, 707, 1201

Wild, W., Harris, A. I., Eckart, A., Genzel, R., Graf, U. U., Jackson, J. M., Russell, A. P. G., \& Stutzki, J. 1992, A\&A, 265, 447

Wright, E. L. 2007, arXiv:0703.640

Younger, J. D., et al. 2007, ApJ, 671, 1531

Younger, J. D., et al. 2009, ApJ, 704, 803 